\title{
Management of opioid use disorders: a national clinical practice guideline
}

\author{
Julie Bruneau MD MSc, Keith Ahamad MD, Marie-Ève Goyer MD MSc, Ginette Poulin MD, \\ Peter Selby MBBS MHSc, Benedikt Fischer PhD, T. Cameron Wild PhD, Evan Wood MD PhD; on behalf of the \\ CIHR Canadian Research Initiative in Substance Misuse
}

Cite as: CMAJ 2018 March 5;190:E247-57. doi: 10.1503/cmaj.170958

The full guideline in English and French is available in Appendix 1 at www.cmaj.ca/lookup/suppl/doi:10.1503/cmaj.170958/-/DC1

CMAJ Podcasts: interview in English at https://soundcloud.com/cmajpodcasts/170958-guide-eng; entrevue en français au https:// soundcloud.com/cmajpodcasts/170958-guide-fre

See related article at www.cmaj.ca/lookup/doi/10.1503/cmaj.180209

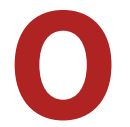

pioid use disorder is one of the most challenging forms of addiction facing the Canadian health care system, and a major contributor to the marked rises in opioidrelated morbidity and death that Canada has been seeing in recent years. The evolving landscape of nonmedical opioid use has become increasingly dominated by prescription opioids diverted from the medical system and, more recently, by highly potent, illicitly manufactured synthetic opioids (e.g., fentanyl and its analogues, including carfentanil). ${ }^{1}$

The mean national rate of hospital admissions related to opioid poisonings increased from 9 hospital admissions per day in $2007 / 08$ to more than 13 admissions per day in $2014 / 15 .{ }^{2}$ A corresponding rise in injection of prescription opioids has been observed among people who inject drugs in Canada, ${ }^{3,4}$ and has been associated with an increased risk of hepatitis $\mathrm{C}$ and HIV infections. $^{5-7}$ For 2016, the mean rate of apparent opioid-related overdose deaths has reached 7.9 per 100000 population (i.e., corresponding to a total of 2861 fatalities), with the highest death rates reported for western Canada. ${ }^{8}$ This upsurge in opioid-related harms, including overdose deaths, ${ }^{2-6,8,9}$ underscores the critical need for coordinated, evidence-based approaches to prevention, treatment and harm reduction to address this national public health emergency.

In most Canadian jurisdictions, poor geographic coverage and availability of evidence-based treatments for substance use disorders has limited the therapeutic options for individuals with opioid use disorder. ${ }^{10}$ Further, even in settings where multiple treatment options are offered, detailed clinical guidance articulating their optimal use for varying presentations of opioid use disorder is lacking. Therefore, this guideline is intended to promote the use of evidence-based interventions for treatment of opioid use disorder across the addiction care continuum in Canada.

\section{KEY POINTS}

- Opioid use disorder is often a chronic, relapsing condition associated with increased morbidity and death; however, with appropriate treatment and follow-up, individuals can reach sustained long-term remission.

- This guideline strongly recommends opioid agonist treatment with buprenorphine-naloxone as the preferred first-line treatment when possible, because of buprenorphine's multiple advantages, which include a superior safety profile in terms of overdose risk.

- Withdrawal management alone is not recommended, because this approach has been associated with elevated risks (e.g., syringe sharing) and death from overdose in comparison to providing no treatment, and high rates of relapse when implemented without immediate transition to long-term evidence-based treatment.

- This guideline supports using a stepped and integrated care approach, in which treatment intensity is continually adjusted to accommodate individual patient needs and circumstances over time, and recognizes that many individuals may benefit from the ability to move between treatments.

\section{Scope}

This guideline was developed to provide Canadian health professionals with an educational tool and clinical practice recommendations for the treatment of opioid use disorder. These recommendations are primarily relevant for the clinical management of this disorder in adults, including young adults.

This guideline is intended for use by physicians and nurse practitioners, allied health care providers, pharmacists, medical educators and clinical care case managers, with or without specialized experience in addiction treatment. Such guidelines are the main resources to inform policy-makers and health care administrators 
at the provincial, territorial and national levels for the development of evidence-based strategies. This guideline is intended to serve as a tool to address current gaps in care for opioid use disorder, addiction-medicine training for clinicians and other health care professionals, and treatment access policies across the country.

The recommendations in this guideline are based on the clinical evidence base regarding treatment approaches for opioid use disorder currently available in Canada, including oral opioid agonist treatment and antagonist pharmacotherapies, as well as withdrawal management strategies, residential treatment and psychosocial treatment interventions. The evidence base for pharmacotherapies not yet widely available in Canada, including long-acting and extended-release opioid antagonists, as well as injectable opioid agonist treatment (i.e., diacetylmorphine and hydromorphone), was not reviewed in this guideline.

\section{Methods}

The Canadian Research Initiative in Substance Misuse (CRISM), a Canadian Institutes of Health Research (CIHR)-funded research network composed of four regional networks (nodes) distributed across Canada (British Columbia, the Prairies, Ontario and Quebec-Atlantic), developed this national guideline using a structured literature review approach. Relevant search terms and structured search strategies were used to search PubMed, Web of Science, the Cochrane Library databases and reference lists using a hierarchical approach, whereby meta-analyses and systematic reviews were given the most weight, followed by individual randomized controlled trials (RCTs), quasi-experimental studies, observational studies and, lastly, expert opinion. At least two independent CRISM staff members manually reviewed titles, abstracts and full text of identified citations, selected evidence for inclusion, and compiled narrative evidence reviews for the guideline review panel. A detailed description of the methods used to compile evidence summaries for each recommendation can be found in Appendices 1 and 2, available at www.cmaj.ca/ lookup/suppl/doi:10.1503/cmaj.170958/-/DC1.

\section{Composition of guideline review panel}

Each of the four CRISM nodes nominated a clinical lead, to whom the coordination of guideline review activities was delegated in each region. In consultation with the node principal investigator, relevant individual experts and stakeholder organizations from their region, each clinical lead invited 7-13 individuals to participate on the review committee. Including the clinical leads and principal investigators, the pan-Canadian review committee consisted of 43 individuals, including primary care physicians, addiction medicine physicians and other specialists, nurse practitioners and registered nurses, social workers, pharmacists, program managers and administrators, and policy-makers.

\section{Development of recommendations}

Recommendations were developed and graded using the Grading of Recommendations Assessment, Development and Evaluation (GRADE) tool ${ }^{11-14}$ (Box 1) through an iterative consensus process. The node principal investigators developed draft recommenda- tions and assigned initial GRADE scores, which were then revised by the full committee in two consecutive rounds of review, as described below. Following each round of review, two guideline authors (E.W. and J.B.) edited and approved the final version of recommendations, which incorporated all input received from committee members, for subsequent committee review (Appendices 1 and 2 provide a more detailed explanation of the evidence underlying each recommendation and score).

\section{Review of recommendations}

The review process consisted of two rounds of revisions of the draft guideline recommendations and evidence review by the panCanadian review committee. CRISM staff consolidated guideline revisions and conducted additional structured literature searches as needed to address committee feedback. Differences in opinion or interpretation with regard to the guideline recommendations or the evidence review were resolved through facilitated discussions in regional committee teleconferences or direct communication. A final decision was reached for all cases without the need for arbitration. Following the two rounds of committee review, two international experts and two organizations representing people affected by opioid use disorders reviewed and provided input on the final draft.

\section{Management of competing interests}

This guideline was entirely funded through the CIHR-funded CRISM network and without pharmaceutical industry support. Competing interests were assessed using the Guidelines International Network's Principles for Disclosure of Interests and Management of Conflicts in Guidelines. ${ }^{15}$ No current or ongoing direct competing interests were disclosed by the 43 members of the review committee or the four CRISM principal investigators on screening for participation in the review committee. Among the 43 committee members, five individuals reported past direct competing interests in the five years before committee participation in the form of paid consulting, services as a technical advisor, or fees to participate in a speaker panel or training seminar. To mitigate potential, perceived or real risk of bias, these five individuals were asked to recuse themselves from the final review and approval process, which included formal endorsement of the guideline recommendations.

\section{Recommendations}

The complete guideline is available in Appendix 1 and includes a detailed discussion of the evidence pertaining to the 11 recommendations presented in Table 1 . In this synopsis, we briefly review the evidence base supporting selected key recommendations.

\section{First- and second-line treatment options}

Initiate opioid agonist treatment (with buprenorphine-naloxone whenever feasible), to reduce the risk of toxicity, morbidity and death, and to facilitate safer take-home dosing (strong recommendation; high-quality evidence).

For individuals responding poorly to buprenorphine-naloxone, consider transition to methadone treatment (strong recommendation; high-quality evidence). 
Initiate opioid agonist treatment with methadone when treatment with buprenorphine-naloxone is not the preferred option (strong recommendation; high-quality evidence).

Given the superior safety profile of buprenorphine-naloxone and its potential for flexible take-home dosing in comparison to other opioid agonist medications, ${ }^{16-20}$ we strongly recommend initiating opioid agonist treatment with buprenorphine-naloxone as the preferred first-line treatment when possible. Alternatively, methadone should be considered for individuals poorly responding to buprenorphine-naloxone, or when buprenorphine-naloxone is not the preferred option. ${ }^{18,21}$ The main advantages and disadvantages of methadone and buprenorphine-naloxone are summarized in Table 2.

Compared with use of $\alpha_{2}$-adrenergic agonists or psychosocial treatment alone, opioid agonist treatment with buprenorphinenaloxone or methadone has proven superior in terms of reten- tion in treatment, sustained abstinence from illicit opioid use, and reduced risk of morbidity and death..$^{21,25,37-40}$ Recent metaanalyses have found that buprenorphine-naloxone and methadone were essentially equally efficacious across these traditional metrics when sufficient (i.e., medium- or high-dose, but not low-dose) buprenorphine-naloxone or methadone were used. ${ }^{18,21,38}$

\section{Risk of death}

While shown to be essentially as efficacious as methadone in clinical trials, ${ }^{18,21,38}$ the partial opioid agonist buprenorphine-naloxone has several safety advantages over methadone (a full opioid agonist), including a reduced risk of fatal overdose because of its lower potential for respiratory depression. ${ }^{16,17,19,25}$ According to a large retrospective study conducted in the United Kingdom, which included more than 19 million prescriptions over a six-year period

\section{Box 1: GRADE approach and interpretation of grading}

The GRADE approach ${ }^{11-14}$ to rating quality of evidence starts with a simplified categorization of study types (i.e., meta-analyses and RCTs, quasiexperimental studies, observational studies and expert opinion), accompanied by initial estimated levels of confidence (i.e., high, moderate, low or very low) in the estimate of a treatment effect. The rating scheme allows for factors that would raise or lower a level of confidence. Factors that would lower confidence in evidence include risk of bias, inconsistency across the RCTs, indirectness and publication bias; factors that would increase confidence include large effect size and an observed dose-response effect. The final quality ratings are reflective of the confidence in the estimated effect in context of bias and limitations that have been identified, as described below:

- High: We are very confident that the true effect lies close to that of the estimate of the effect.

- Moderate: We are moderately confident in the effect estimate. The true effect is likely to be close to the estimate of the effect, but there is a possibility that it is substantially different.

- Low: Our confidence in the effect estimate is limited. The true effect may be substantially different from the estimate of the effect.

- Very low: We have very little confidence in the effect estimate. The true effect is likely to be substantially different from the estimate of effect.

The GRADE approach uses a binary system to classify strength of recommendations as strong or weak. It is important to note that although quality of evidence is an important factor when classifying strength of recommendations, "strong" or "weak" in this case does not refer exclusively to the quality of evidence underlying a given recommendation. Except for cost and resource allocation, the recommended GRADE factors to classify strength of recommendations were considered:

- Balance between desirable and undesirable effects: The larger the difference between the desirable and undesirable effects, the higher the likelihood that a strong recommendation is warranted. The narrower the gradient, the higher the likelihood that a weak recommendation is warranted.

- Quality of evidence: The higher the quality of evidence, the higher the likelihood that a strong recommendation is warranted.

- Values and preferences: The more values and preferences vary, or the greater the uncertainty in values and preferences, the higher the likelihood that a weak recommendation is warranted.

\section{Interpretation of strength of recommendations}

Examples of how a strong versus weak recommendation could be interpreted by selected audience or user groups are listed below.

A strong recommendation indicates the following:

- For patients: Most people in your situation would want the recommended course of action, and only a small proportion would not; you should request discussion with your care provider if the intervention is not offered.

- For clinicians: Most patients should receive the recommended course of action. As an example, in this scenario, an algorithm or decision-making tool would not be necessary - the benefits of the recommended course of action would clearly outweigh any advantages of alternative interventions.

- For health care administrators: The recommendation can be adopted as a policy in most situations.

A weak recommendation indicates the following:

- For patients: Most people in your situation would want the recommended course of action, but many would not.

- For clinicians: You should recognize that different choices will be appropriate for different patients and that you must help each patient to arrive at a management decision consistent with her or his values and preferences. In this scenario, an algorithm or decision-making tool would be advantageous to determine the best course of action.

- For health care administrators: Policy-making will require substantial debate and involvement of many stakeholders.

Note: GRADE = Grading of Recommendations Assessment, Development and Evaluation; RCT = randomized controlled trial. 
(2007-2012), buprenorphine-naloxone was six times safer than methadone in terms of overdose risk. ${ }^{19}$ In a similar study conducted in France between 1994 and 1998, the annual estimated death rate related to methadone was at least three times greater than that for buprenorphine. ${ }^{16}$ More recently, a systematic review and meta-analysis of observational cohort studies confirmed that pooled death rates were lower for buprenorphine-naloxone compared with methadone, both during treatment and in the period

Table 1: Summary of recommendations for the clinical management of opioid use disorder*

Recommendation

Quality of evidence

Strength

of recommendation

First- and second-line treatment options

1. Initiate opioid agonist treatment with buprenorphine-naloxone whenever feasible, to reduce the risk of toxicity, morbidity and death, and to facilitate safer take-home dosing.

2. For individuals responding poorly to buprenorphine-naloxone, consider transition to methadone treatment.

3. Initiate opioid agonist treatment with methadone when treatment with buprenorphine-naloxone is not the preferred option.

4. For individuals with a successful and sustained response to methadone who express a desire for treatment simplification, consider transition to buprenorphine-naloxone, because its superior safety profile allows for more routine take-home dosing and less frequent medical appointments.

\section{Alternative or adjunct treatment options}

5. In patients for whom first- and second-line treatment options are ineffective or contraindicated, opioid agonist treatment with slow-release oral morphine (initially prescribed as once-daily witnessed doses) can be considered. Slow-release oral morphine treatment should be prescribed only by physicians with a Section 56 exemption to prescribe methadone, or following consultation with an addiction practitioner experienced in opioid agonist treatment with slow-release oral morphine.

6. Offering withdrawal management alone (i.e., detoxification without immediate transition to long-term addiction treatment $\dagger$ ) should be avoided, because this approach has been associated with increased rates of relapse, morbidity and death.

7. When withdrawal management (without transition to opioid agonist treatment) is pursued, provide supervised slow (>1 mo) opioid agonist taper (in an outpatient or residential treatment setting) rather than a rapid (<1 wk) taper. During opioid-assisted withdrawal management, patients should be transitioned to long-term addiction treatment $†$ to help prevent relapse and associated health risks.

8. For patients with a successful and sustained response to opioid agonist treatment who wish to discontinue treatment (i.e., desiring medication cessation), consider a slow taper approach (over months to years, depending on the patient). Ongoing addiction care should be considered on cessation of opioid use.

9. Psychosocial treatment interventions and supports should be routinely offered but should not be viewed as a mandatory requirement for accessing opioid agonist treatment.

10. Oral naltrexone can also be considered as an adjunct medication if cessation of opioid use is achieved.

\section{Adjunct harm-reduction strategies}

11. Information and referrals to take-home naloxone programs and other harm reduction services (e.g., provision of clean drug paraphernalia), as well as other general health care services, should be routinely offered as part of standard care for opioid use disorders.

Moderate

Strong

Moderate Strong

Moderate Strong

Moderate Strong

Low

Weak

Moderate Strong

*The evidence supporting these recommendations is discussed in detail in Appendix 1.

†Long-term addiction treatment: In this context, "addiction treatment" refers to continued care for opioid use disorder delivered by an experienced care provider, which could include pharmacologic treatment (opioid agonist treatment or antagonist treatment), evidence-based psychosocial treatment, residential treatment or combinations of these treatment options. In isolation, withdrawal management, harm reduction services, low-barrier housing and unstructured peer-based support would not be considered "addiction treatment." Opioid agonist treatment may be provided in an outpatient or in an inpatient addiction-treatment setting. 
after completing treatment. ${ }^{41} \mathrm{~A}$ retrospective analysis of administrative mortality data in British Columbia showed that among the 1674 deaths associated with prescription opioids reported between 2004 and 2013, 25\% involved methadone without another prescription opioid, 5\% involved methadone and another prescription opioid, and the remaining $70 \%$ involved all other nonmethadone prescription opioids and heroin (no specific data on buprenorphine-naloxone). ${ }^{42}$ Overall, the safety advantages of buprenorphine-naloxone are also relevant from a public health perspective; if diverted to an individual other than to whom it was prescribed, it is far less likely than methadone to cause overdoserelated harms, including death.

\section{Drug-drug interactions and adverse events}

Methadone is associated with a higher frequency of clinically important adverse reactions and drug-drug interactions (e.g.,

\section{Table 2: Advantages and disadvantages of methadone versus buprenorphine-naloxone}

Methadone

\section{Advantages}

- Potentially better treatment retention, particularly in patients with higher-intensity opioid use disorder (e.g., long history of opioid use, injection heroin use, high tolerance and frequent use), or at high risk of dropping out ${ }^{18,22,23}$

- May be more effective for withdrawal-symptom control in chronic, severe opioid use disorder 22,23

- Treatment initiation may be easier

- No maximum dose

- Approved in Canada for the indication of pain control

\section{Buprenorphine-naloxone}

- Health Canada exemption is not required to prescribe buprenorphine-naloxone in most provinces and territories (Appendix 1)

- Lower risk of overdose due to partial agonist properties and ceiling effect for respiratory depression (in the absence of benzodiazepines or alcohol) ${ }^{19,24,25}$

- Lower risk of public safety harms if diverted ${ }^{26,27}$

- Milder adverse effect profile 22,23

- Easier to transition from buprenorphine-naloxone to methadone if treatment is unsuccessful ${ }^{22,23}$

- Shorter time to achieve therapeutic dose $(1-3 \mathrm{~d})^{28-30}$

- Lower risk of toxicity and drug-drug interactions ${ }^{31}$

- Milder withdrawal symptoms when discontinuing treatment; may be a better option for individuals with lower-intensity opioid dependence (e.g., oral opioid dependence, infrequent or no injection use, short history of opioid use disorder), and individuals planning to taper off opioid agonist treatment in a relatively short period $^{22,23}$

- Optimal for rural and remote locations where access to care is limited, methadone prescribers are lacking, or daily witnessed ingestion at a pharmacy is not feasible

- More flexible dosing schedules (e.g., alternate-day dosing, earlier provision of 1- to 2-week take-home prescriptions, and unobserved home inductions) support patient autonomy and can reduce costs $^{32-35}$

- Easier to adjust and retitrate following missed doses, owing to its partial agonist properties

- Potentially lower treatment retention, particularly in higherintensity opioid use disorder with low-dose buprenorphinenaloxone ${ }^{18}$

- May cause precipitated withdrawal if appropriate dose-induction protocols are not followed ${ }^{30}$

- Suppression of withdrawal symptoms may be inadequate for individuals with high opioid tolerance ${ }^{22,23}$

- Reversing effects of overdose can be challenging because of the pharmacology of buprenorphine (i.e., high affinity for opioid receptors and long half-life) ${ }^{31}$

- Patients require education on how to take sublingual doses correctly (i.e., hold under tongue until dissolved - up to 10 minutes; do not drink or smoke, and minimize swallowing)

- Nonadherence to treatment may require frequent reinductions 
with antiretrovirals, antibiotics and some antidepressants) than buprenorphine-naloxone. ${ }^{43-45}$

Regarding adverse events, only a minor effect on corrected QT (QTC) interval duration has been reported with therapeutic doses of buprenorphine-naloxone, without proarrhythmic effects. ${ }^{46,47}$ Conversely, methadone can increase the risk of a rare but fatal ventricular arrhythmia (torsades de pointes) because of its substantial QT-prolonging effects, especially at higher doses. ${ }^{47}$ Case series have reported that transitioning patients taking methadone who were experiencing torsades de pointes to buprenorphine corrected the condition. ${ }^{48,49}$

Long-term opioid use, including opioid agonist treatment, may lead to abnormalities in the endocrine system, mainly affecting the gonadal axis and leading to hypogonadism. ${ }^{50,51} \mathrm{~A}$ meta-analysis including four studies showed that methadone was associated with a significantly higher frequency of male sexual dysfunction than buprenorphine-naloxone. ${ }^{52}$

\section{Treatment flexibility}

For methadone, to optimize patient and public safety and prevent diversion, provincial and territorial clinical practice guidelines generally recommend a slow increase in dosing and a mandatory period of daily witnessed ingestion at pharmacies following treatment initiation (range 2-3 mo), which should be continued indefinitely in patients with ongoing substance use or other measures of clinical and social instability. Daily pharmacy attendance can cause substantial lifestyle (e.g., employment) disruption to patients. In contrast, because of comparative safety advantages, buprenorphine-naloxone can safely be provided for take-home dosing as soon as clinical stability is achieved (e.g., often within 7-10 days of treatment initiation), which has the potential to improve patients' quality of life and reduce the burden of requiring daily pharmacy attendance without compromising retention in care. ${ }^{32,53,54}$ Further, the partial agonist effect allows more rapid titration to a therapeutic dose $(<1 \mathrm{wk}) .^{22}$

There is also potential for cost savings and greater patient autonomy with home induction of buprenorphine-naloxone, ${ }^{34,35}$ which under appropriate circumstances, yields similar outcomes as office-based induction in terms of patient safety, retention and reductions in nonmedical opioid use. ${ }^{33}$ However, buprenorphinenaloxone requires an individual to be in moderate withdrawal before induction to avoid precipitated withdrawal, which can present greater challenges than methadone inductions, making buprenorphine-naloxone less attractive in certain cases. ${ }^{55}$

The relative ease of transition from buprenorphine-naloxone to methadone further supports the use of buprenorphinenaloxone as the preferred first-line treatment when appropriate. A stepped care strategy involving initial treatment with buprenorphine-naloxone and transition to methadone if necessary was shown to be equally efficacious as an optimally delivered methadone treatment. ${ }^{56}$ In contrast, although transitioning to buprenorphine-naloxone from methadone is achievable, ${ }^{57}$ this practice must be individually tailored and can be highly challenging for some patients..$^{58}$ Indeed, when transitioning from higher daily doses of methadone, there is an increased risk of substantial withdrawal symptoms and consequent relapse; adjunct medications or inpatient treatment (e.g., medically supervised withdrawal management programs) may be required for safe conversion in such cases. ${ }^{57}$

\section{Alternative specialist-led approach}

In patients for whom first-and second-line treatment options are ineffective or contraindicated, opioid agonist treatment with slow-release oral morphine (initially prescribed as once-daily witnessed doses) can be considered. Slow-release oral morphine treatment should be prescribed only by physicians with a Section 56 exemption to prescribe methadone, or after consultation with an addiction practitioner with experience in opioid agonist treatment with slow-release oral morphine (strong recommendation; moderate-quality evidence).

We recommend the 24-hour formulation of slow-release oral morphine as a potential option for opioid agonist treatment in cases where both buprenorphine-naloxone and methadone are ineffective or contraindicated. Other formulations of oral morphine, such as twice-daily, 12-hour sustained- or extended-release formulations - or any other long-acting synthetic opioid - have not been empirically studied in this context and are not recommended by this committee for the treatment of opioid use disorder.

A 2013 Cochrane review including three RCTs comparing slow-release oral morphine to methadone (two studies) or buprenorphine-naloxone (one study) reported no significant difference between treatments for retention in treatment, medication adherence or nonmedical opioid use. ${ }^{59}$ Since this review, an international multisite randomized cross-over trial found that slow-release oral morphine was as effective as methadone in reducing illicit opioid use and retaining individuals in treatment, ${ }^{60}$ and superior to methadone for overall patient satisfaction, and reducing cravings and symptoms of dysthymia. ${ }^{61-63}$ However, because of the small number of trials comparing slowrelease oral morphine to other opioid agonist treatments, slowrelease oral morphine should generally be considered only for use in patients who are intolerant to or have not responded to first- and second-line opioid agonist treatment, and who remain at high risk of opioid-related harms, including overdose death.

Widely used for opioid agonist treatment in Europe, slowrelease oral morphine has been eligible for coverage under Health Canada's Non-Insured Health Benefits program for the treatment of opioid use disorder since November $2014,{ }^{64}$ although this indication is considered off-label in Canada. It is the consensus of this committee that health care providers who wish to prescribe opioid agonist treatment based on slow-release oral morphine should hold a valid federal exemption under Section 56 of the Controlled Drugs and Substances Act to prescribe methadone as an indication of experience with opioid use disorder treatment, or consult with an addiction medicine specialist experienced in prescribing slow-release oral morphine for this indication.

Considering the wide range of doses described in the literature, ${ }^{65}$ we suggest that practitioners holding a federal exemption with no prior experience in prescribing slow-release oral morphine for opioid agonist treatment also consult with a specialist. As is the case with methadone, because of patient and public safety risks associated with slow-release oral morphine diversion, 
the committee suggests that the standard should be to prescribe slow-release oral morphine as daily witnessed doses.

As in the case of methadone, take-home doses of slow-release oral morphine should be prescribed only to individuals who meet recommended criteria (Appendix 1). If take-home doses of slow-release oral morphine are prescribed, individuals should be closely monitored and appropriate strategies should be employed to reduce the risk of diversion (e.g., random urine drug testing, unannounced pill counts).

\section{Approach to avoid}

Offering withdrawal management alone (i.e., detoxification without immediate transition to long-term addiction treatment) should be avoided, because this approach has been associated with increased rates of relapse, morbidity and death (strong recommendation; moderate-quality evidence).

This guideline strongly recommends against a treatment strategy involving withdrawal management alone. Moderate-quality evidence indicates that this approach, when administered without linkage to long-term addiction treatment and care, is associated with elevated risk of relapse, HIV and hepatitis $C$ transmission, and death from drug overdose. ${ }^{66-68}$ Patients should be clearly informed of the known risks of withdrawal management alone and engaged in discussion about other treatment options corresponding to their individual needs and circumstances.

Studies have found no significant difference among buprenorphine-naloxone, methadone and $\alpha_{2}$-adrenergic agonists in terms of severity of withdrawal symptoms, adverse effects, withdrawal completion and, importantly, of the poor sustained abstinence rates in the absence of linkage to long-term addiction treatment. ${ }^{69-71}$ If withdrawal management is offered as part of an immediate transition to psychosocial or residential treatment, then, among available pharmacologic approaches used for withdrawal management, a buprenorphine-naloxone taper may offer advantages such as faster symptom relief and higher rates of withdrawal completion over methadone tapers. ${ }^{71}$ If cessation of opioid use is achieved, oral naltrexone can be considered as an adjunct medication to support abstinence (low-quality evidence). ${ }^{72}$

\section{Adjunct psychosocial treatment interventions}

Psychosocial treatment interventions and supports should be routinely offered in conjunction with pharmacologic treatment, but should not be viewed as a mandatory requirement for accessing opioid agonist treatment (strong recommendation; moderatequality evidence).

In 2011, a Cochrane review of 35 RCTs compared the effect of adding psychosocial treatment interventions to standard opioid agonist treatment programs that included clinician-led medical management and counselling. ${ }^{73}$ The authors found, based on moderate- and high-quality evidence, that ancillary psychosocial treatment did not confer additional benefits in terms of retaining individuals in treatment, supporting abstinence or preventing relapse. Clinical trials published after this review have yielded mixed results (Appendix 1). Collectively, these findings suggest that while information and referrals to psychosocial treatment interventions and supports should be routinely offered, a patient's decision not to participate in psychosocial treatment interventions should never preclude or delay provision of evidence-based pharmacologic treatments. ${ }^{73}$ Considering the apparent gaps in knowledge existing in this area, and also that psychosocial treatment interventions and peer-based support systems without associated opioid agonist treatment are widely used throughout Canada's addiction treatment services, the CRISM network is currently undertaking a comprehensive review of the role of psychosocial treatment interventions and other supports in the care of people with opioid use disorder.

\section{Values and preferences}

Inclusion of values and preferences for balancing the magnitude of desirable and undesirable outcomes in management of opioid use disorder was based on relevant published literature, including studies of patient values and preferences, on the expertise of our review panel, and on consultations with groups of experienced clinicians and two community groups of people affected by opioid use disorders. Based on this global assessment, this guideline recommends using a stepped and integrated care approach, in which treatment intensity is continually adjusted to accommodate individual patient needs and circumstances over time, and recognizes that many individuals may benefit from the ability to move between treatments (Figure 1). ${ }^{74}$ The choice of treatment should also take into account several patient-specific factors such as initial presentation, comorbidities (e.g., advanced liver disease, prolonged QTc interval), drug-drug interactions, treatment preference and response to treatment, and the prescriber's experience. Details of value- and preference-specific assessments according to recommendations can be found in Appendices 1 and 2 .

\section{Special considerations}

\section{Pregnant women}

Opioid agonist treatment has long been the standard therapy for opioid use disorder in pregnant women. ${ }^{75,76}$ Abundant supporting evidence has rendered methadone the most frequently prescribed opioid agonist during pregnancy; however, more recent research suggests that buprenorphine (monoproduct) may be similarly safe and effective for the treatment of opioid use disorder in pregnant women. ${ }^{75,77-79}$ We suggest that care providers seek specialist consultation as needed to determine, on a case-by-case basis, the appropriate agent for opioid agonist treatment of a pregnant patient. Withdrawal management is not suggested during pregnancy, primarily because of the high rates of relapse and the adverse outcomes associated with rapid withdrawal and subsequent relapse, such as maternal and fetal distress, fetal death, fetal hypoxia, preterm labour and long-term developmental issues. ${ }^{80}$

\section{Residential treatment}

There are no well-controlled clinical trials or meta-analyses comparing the efficacy of residential treatment to other treatment interventions for opioid use disorder. Observational cohort studies have found that relapse to nonmedical opioid use is relatively common following residential treatment provided without concomitant opioid agonist treatment, with reported relapse rates 


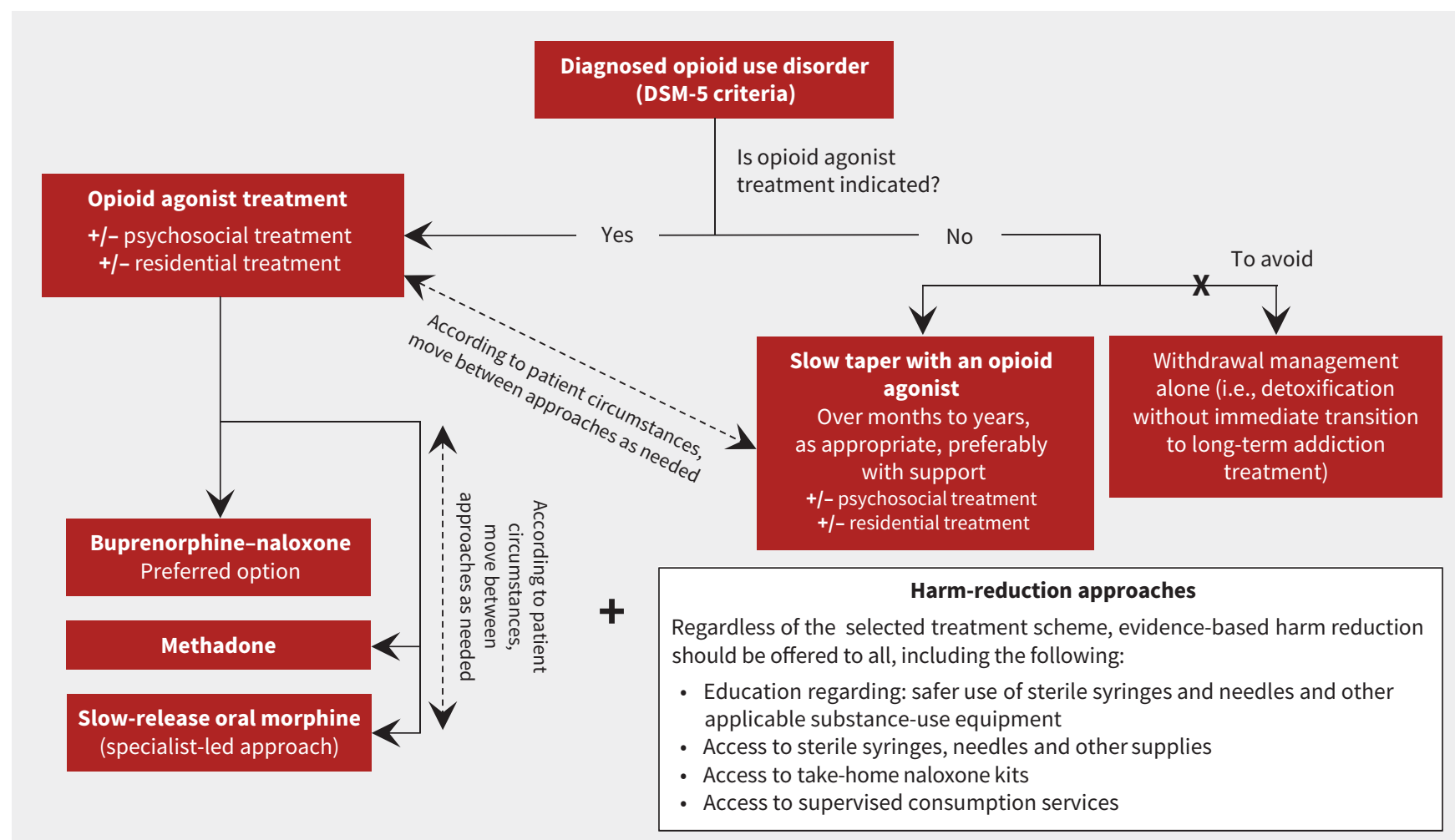

Figure 1: Treatment algorithm for opioid use disorder. Note: A particular medication may not be indicated for a variety of reasons, including ineffectiveness or medical contraindication, comorbidities, drug-drug interactions, patient preference and specific circumstances, and prescriber's experience. Any treatment for opioid use disorder, but particularly slow agonist tapers, should incorporate evidence-based psychosocial interventions with qualified professionals, motivational interviewing, long-term monitoring of substance use, provision of comprehensive primary care, and referrals to psychosocial treatment interventions and psychosocial supports as appropriate, with specialist care as required, to optimize physical and mental wellness as the patient progresses in recovery. Withdrawal management (formerly "detoxification") without linkage to long-term addiction treatment is to be avoided, and patients desiring such approach should be informed of risks and encouraged toward other treatment options that would suit their circumstances. DSM-5 = Diagnostic and Statistical Manual of Mental Disorders, fifth edition. ${ }^{74}$

ranging from $60 \%$ to $90 \%$, potentially increasing the risk of morbidity and death. ${ }^{20,81,82}$

In some residential treatment settings, provision of opioid agonist treatment has been viewed as incompatible with an abstinence-oriented approach, causing the two treatment models to develop and operate separately from one another. ${ }^{83}$ Over time, the proven benefits of opioid agonist treatment have prompted efforts to integrate these approaches, and some residential treatment programs have revised admission policies and service provision to accommodate evidence-based treatment and patients' preference. ${ }^{83-85}$ Given the known benefits of opioid agonist treatment, priority should be given to programs and initiatives aimed at strengthening both the opioid agonist and residential treatment systems of care through an integration of evidence-based treatment approaches to opioid use disorder.

\section{Implementation}

Considering that research evidence for treatment of opioid use disorder is limited for youth, older adults and other populations (e.g., individuals living with concurrent chronic pain, pregnant women, incarcerated individuals and Indigenous populations), care provision for these populations may require adjustments in terms of models of care, service delivery and intensity, or coverage policies at the provincial and territorial level. It is also noted that much of the available research evidence in this area involved patients with moderate to severe opioid use disorder as per the Diagnostic and Statistical Manual of Mental Disorders, fifth edition. ${ }^{74}$

In most Canadian jurisdictions, the lack of resources dedicated to health care provider education and to overall addiction care, as well as the absence of comprehensive provincial and territorial or national guidelines, have delayed the implementation of evidence-based treatment strategies for opioid use disorder across the addiction care continuum. In the future, updates to this guideline and, where appropriate, modifications to guideline recommendations, will follow the publication of novel research evidence. The CRISM network is developing a systematic knowledge transfer process to support the implementation and uptake of this national guideline in collaboration with key provincial regulatory agencies and front-line clinicians.

\section{Other guidelines}

Two main guidelines on treatment of opioid use disorder were published in the past decade, one by the American Society of Addiction Medicine ${ }^{86}$ and the other by the World Health Organization..$^{87} \mathrm{~A}$ key 
structural feature that distinguishes the current guideline from these previous guidelines is its presentation of treatment options as components of a continuum of care, providing one comprehensive set of recommendations to facilitate individualized treatment selection. Whereas earlier guidelines present the evidence and recommendations pertaining to each intervention separately, this guideline stratifies available interventions in relation to each other in terms of comparative safety, effectiveness and intensity, as well as discussing interventions to avoid and considerations for moving between and combining treatments.

Although this guideline's endorsement of opioid agonist treatment is in line with other recent international guidelines, its recommendation of buprenorphine-naloxone as a preferred first-line medication is novel. This guideline is also distinct in its explicit classification of standalone withdrawal management as an approach to avoid. However, like other contemporary guidelines, this guideline emphasizes the importance of collaborative patient-centred care that accommodates patient preferences.

In terms of Canadian guidance for the clinical management of opioid use disorder, existing provincial guidelines for opioid agonist treatment are generally focused on specific dosing recommendations, medical visit schedules and treatment plans, but do not recommend approaches across the spectrum of addiction care, nor first- versus second-line treatments or strategies to avoid (e.g., withdrawal management alone). Therefore, unlike available provincial guidelines, this national guideline proposes a set of high-level recommendations for the clinical management of opioid use disorders to inform physicians about a broad range of available treatment options.

\section{Gaps in knowledge}

Whereas there is a need for further research substantiating the evidence base specific to individuals with prescription opioid use disorder, current evidence comparing methadone and buprenorphine-naloxone suggests that both treatments appear equally efficacious, with the above-mentioned advantages of buprenorphine-naloxone also relevant in the prescription opioid context. ${ }^{88}$ In addition, as with patients who primarily inject illicit opioids (e.g., heroin), existing research shows that most patients with a prescription opioid use disorder will relapse to nonmedical opioid use when opioid agonist treatment is used for short periods or tapered. ${ }^{89,90}$ Additional research is required to identify the ideal length of treatment with opioid agonists and optimal tapering strategies for individuals who have achieved long-term remission and wish to discontinue opioid agonist treatment, as well as to assess the efficacy of nonpharmacotherapy treatment or intervention options, including residential treatment.

Finally, given the substantial need to expand treatment options nationally, major emphasis among policy-makers is required to gain a better understanding and address barriers to evidence-based treatments, as reviewed in this guideline. Urgent action is required at multiple levels to reduce barriers to accessing specialist-led treatment interventions not reviewed here, such as injectable opioid agonist treatment with diacetylmorphine or hydromorphone.
Individuals with opioid use disorder have many comorbid medical and mental health conditions, and face a range of structural and social difficulties. As such, it is crucial to establish health care implementation science mechanisms to promote action on opioid use disorder on several fronts and monitor the progression of the opioid emergency response across the country in the short- and long-term. To this end, it is paramount to develop a multidisciplinary and actionable care roadmap to improve clinical care strategies (i.e., address wait times for treatment and linkage to care), and strengthen the integration of care and research across the public health and clinical domains.

\section{Conclusion}

Opioid use disorder is a public health emergency nationwide. This guideline recommends strongly against offering withdrawal management in isolation; the resulting loss of tolerance coupled with high rates of relapse associated with this practice increases the risk of overdose death. Instead, this guideline recommends a stepped care approach involving opioid agonist treatment with buprenorphine-naloxone as a first-line treatment, progressing toward methadone as second-line if required; then, if needed and appropriate, toward slow-release oral morphine with the support of a specialist. Furthermore, although out of scope of this guideline, evidence-based harm-reduction approaches such as naloxone programs and novel treatments not yet widely available in Canada must be urgently expanded.

\section{References}

1. Fairbairn N, Coffin PO, Walley AY. Naloxone for heroin, prescription opioid, and illicitly made fentanyl overdoses: challenges and innovations responding to a dynamic epidemic. Int J Drug Policy 2017;46:172-9.

2. Canadian Institute for Health Information, Canadian Centre on Substance Abuse. Hospitalizations and emergency department visits due to opioid poisoning in Canada. Ottawa: Canadian Institute for Health Information; 2016.

3. Bruneau J, Roy E, Arruda N, et al. The rising prevalence of prescription opioid injection and its association with hepatitis $\mathrm{C}$ incidence among street-drug users. Addiction 2012;107:1318-27.

4. Hadland SE, DeBeck K, Kerr T, et al. Prescription opioid injection and risk of hepatitis $C$ in relation to traditional drugs of misuse in a prospective cohort of street youth. BMJ Open 2014;4:e005419.

5. Lankenau SE, Kecojevic A, Silva K. Associations between prescription opioid injection and hepatitis $C$ virus among young injection drug users. Drugs (Abingdon Engl) 2015;22:35-42.

6. Puzhko S, Roy E, Jutras-Aswad D, et al. High hepatitis C incidence in relation to prescription opioid injection and poly-drug use: assessing barriers to hepatitis C prevention. Int J Drug Policy 2017;47:61-8.

7. Hennink M, Abbas Z, Choudhri Y, et al. Risk behaviours for infection with HIV and hepatitis $C$ virus among people who inject drugs in Regina, Saskatchewan. Can Commun Dis Rep 2007;33:53-9.

8. Special Advisory Committee on the Epidemic of Opioid Overdoses. National report: Apparent opioid-related deaths in Canada (January 2016 to June 2017) Ottawa: Public Health Agency of Canada; 2017. Available: www.canada.ca/en/ public-health/services/publications/healthy-living/apparent-opioid-related -deaths-report-2016-2017-december.html (accessed 2018 Feb. 20).

9. A province-by-province look at opioid-overdose stats, including fentanyl. Global News. 2016 Nov. 17. Available: http://globalnews.ca/news/3072316 /a-province-by-province-look-at-opioid-overdose-stats-including-fentanyl (accessed 2017 Mar. 9).

10. Eibl JK, Morin K, Leinonen E, et al. The state of opioid agonist therapy in Canada 20 years after federal oversight. Can J Psychiatry 2017;62:444-50.

11. Andrews JC, Schunemann HJ, Oxman AD, et al. GRADE guidelines: 15. Going from evidence to recommendation-determinants of a recommendation's direction and strength. J Clin Epidemiol 2013;66:726-35. 
12. Alonso-Coello P, Oxman AD, Moberg J, et al. GRADE Evidence to Decision (EtD) frameworks: a systematic and transparent approach to making well informed healthcare choices. 2: Clinical practice guidelines. BMJ 2016;353:i2089.

13. Guyatt GH, Oxman AD, Kunz R, et al. GRADE: going from evidence to recommendations. BMJ 2008;336:1049-51.

14. Guyatt GH, Oxman AD, Kunz R, et al. What is "quality of evidence" and why is it important to clinicians? BMJ 2008;336:995-8

15. Schünemann HJ, Al-Ansary LA, Forland F, et al. Guidelines International Network: principles for disclosure of interests and management of conflicts in guidelines. Ann Intern Med 2015;163:548-53.

16. Auriacombe M, Franques $\mathrm{P}$, Tignol J. Deaths attributable to methadone vs. buprenorphine in France. JAMA 2001;285:45.

17. Luty J, O'Gara C, Sessay M. Is methadone too dangerous for opiate addiction? The case for using a safer alternative, buprenorphine, is strong. BMJ 2005;331: 1352-3.

18. Mattick RP, Breen C, Kimber J, et al. Buprenorphine maintenance versus placebo or methadone maintenance for opioid dependence. Cochrane Database Syst Rev 2014;(2):CD002207.

19. Marteau D, McDonald R, Patel K. The relative risk of fatal poisoning by methadone or buprenorphine within the wider population of England and Wales. BMJ Open 2015;5:e007629.

20. Pierce M, Bird SM, Hickman M, et al. Impact of treatment for opioid dependence on fatal drug-related poisoning: a national cohort study in England. Addiction 2016;111:298-308.

21. Nielsen S, Larance B, Degenhardt L, et al. Opioid agonist treatment for pharmaceutical opioid dependent people. Cochrane Database Syst Rev 2016;(5): CD011117.

22. Maremmani I, Gerra G. Buprenorphine-based regimens and methadone for the medical management of opioid dependence: selecting the appropriate drug for treatment. Am J Addict 2010;19:557-68.

23. Bonhomme J, Shim RS, Gooden R, et al. Opioid addiction and abuse in primary care practice: a comparison of methadone and buprenorphine as treatment options. J Natl Med Assoc 2012;104:342-50.

24. Dasgupta N, Bailey EJ, Cicero T, et al. Post-marketing surveillance of methadone and buprenorphine in the United States. Pain Med 2010;11:1078-91.

25. Bell JR, Butler B, Lawrance A, et al. Comparing overdose mortality associated with methadone and buprenorphine treatment. Drug Alcohol Depend 2009; 104:73-7

26. Maxwell JC, McCance-Katz EF. Indicators of buprenorphine and methadone use and abuse: What do we know? Am J Addict 2010;19:73-88.

27. Lofwall MR, Walsh SL. A review of buprenorphine diversion and misuse: the current evidence base and experiences from around the world. J Addict Med 2014;8:315-26.

28. D'Onofrio G, O'Connor PG, Pantalon MV, et al. Emergency department-initiated buprenorphine/naloxone treatment for opioid dependence: a randomized clinical trial. JAMA 2015;313:1636-44.

29. Liebschutz JM, Crooks D, Herman D, et al. Buprenorphine treatment for hospitalized, opioid-dependent patients: a randomized clinical trial. JAMA Intern Med 2014;174:1369-76.

30. Kraus ML, Alford DP, Kotz MM, et al. Statement of the American Society of Addiction Medicine consensus panel on the use of buprenorphine in officebased treatment of opioid addiction. J Addict Med 2011;5:254-63.

31. Moody DE. Metabolic and toxicological considerations of the opioid replacement therapy and analgesic drugs: methadone and buprenorphine. Expert Opin Drug Metab Toxicol 2013;9:675-97.

32. Holland R, Maskrey V, Swift L, et al. Treatment retention, drug use and social functioning outcomes in those receiving 3 months versus 1 month of supervised opioid maintenance treatment. Results from the Super $\mathrm{C}$ randomized controlled trial. Addiction 2014;109:596-607.

33. Lee JD, Vocci F, Fiellin DA. Unobserved "home" induction onto buprenorphine. J Addict Med 2014;8:299-308.

34. Baser O, Chalk M, Fiellin DA, et al. Cost and utilization outcomes of opioiddependence treatments. Am J Manag Care 2011;17(Suppl 8):S235-48.

35. Barnett PG. Comparison of costs and utilization among buprenorphine and methadone patients. Addiction 2009;104:982-92.

36. Baxter LE Sr, Campbell A, Deshields M, et al. Safe methadone induction and stabilization: report of an expert panel. J Addict Med 2013;7:377-86.

37. Fullerton CA, Kim M, Thomas CP, et al. Medication-assisted treatment with methadone: assessing the evidence. Psychiatr Serv 2014;65:146-57.

38. Thomas CP, Fullerton CA, Kim M, et al. Medication-assisted treatment with buprenorphine: assessing the evidence. Psychiatr Serv 2014;65:158-70.

39. Degenhardt L, Bucello C, Mathers B, et al. Mortality among regular or dependent users of heroin and other opioids: a systematic review and meta-analysis of cohort studies. Addiction 2011;106:32-51.
40. Mattick RP, Breen C, Kimber J, et al. Methadone maintenance therapy versus no opioid replacement therapy for opioid dependence. Cochrane Database Syst Rev 2009;(3):CD002209.

41. Sordo L, Barrio G, Bravo MJ, et al. Mortality risk during and after opioid substitution treatment: systematic review and meta-analysis of cohort studies. BMJ 2017;357:j1550.

42. Gladstone EJ, Smolina K, Morgan SG. Trends and sex differences in prescrip tion opioid deaths in British Columbia, Canada. Inj Prev 2016;22:288-90.

43. Tjagvad C, Skurtveit S, Linnet K, et al. Methadone-related overdose deaths in a liberal opioid maintenance treatment programme. Eur Addict Res 2016;22: 249-58.

44. McCance-Katz EF, Sullivan LE, Nallani S. Drug interactions of clinical importance among the opioids, methadone and buprenorphine, and other frequently prescribed medications: a review. Am J Addict 2010;19:4-16.

45. McCance-Katz EF, Rainey PM, Smith P, et al. Drug interactions between opioids and antiretroviral medications: interaction between methadone, LAAM, and delavirdine. Am J Addict 2006;15:23-34

46. Fareed A, Patil D, Scheinberg K, et al. Comparison of QTc interval prolongation for patients in methadone versus buprenorphine maintenance treatment: a 5-year follow-up. J Addict Dis 2013;32:244-51.

47. Alinejad S, Kazemi T, Zamani N, et al. A systematic review of the cardiotoxicity of methadone. EXCLI J 2015;14:577-600.

48. Krantz MJ, Garcia JA, Mehler PS. Effects of buprenorphine on cardiac repolarization in a patient with methadone-related torsade de pointes. Pharmacotherapy 2005;25:611-4.

49. Thanavaro KL, Thanavaro JL. Methadone-induced torsades de pointes: a twist of fate. Heart Lung 2011;40:448-53.

50. Hallinan R, Byrne A, Agho K, et al. Hypogonadism in men receiving methadone and buprenorphine maintenance treatment. Int J Androl 2009;32:131-9.

51. Merza Z. Chronic use of opioids and the endocrine system. Horm Metab Res 2010;42:621-6.

52. Yee A, Loh HS, Hisham Hashim HM, et al. Clinical factors associated with sexual dysfunction among men in methadone maintenance treatment and buprenorphine maintenance treatment: a meta-analysis study. Int J Impot Res 2014;26: 161-6.

53. Amass L, Kamien JB, Mikulich SK. Thrice-weekly supervised dosing with the combination buprenorphine-naloxone tablet is preferred to daily supervised dosing by opioid-dependent humans. Drug Alcohol Depend 2001;61:173-81.

54. Marsch LA, Moore SK, Borodovsky JT, et al. A randomized controlled trial of buprenorphine taper duration among opioid-dependent adolescents and young adults. Addiction 2016;111:1406-15

55. Rosado J, Walsh SL, Bigelow GE, et al. Sublingual buprenorphine/naloxone precipitated withdrawal in subjects maintained on $100 \mathrm{mg}$ of daily methadone. Drug Alcohol Depend 2007;90:261-9.

56. Kakko J, Gronbladh L, Svanborg KD, et al. A stepped care strategy using buprenorphine and methadone versus conventional methadone maintenance in heroin dependence: a randomized controlled trial. Am J Psychiatry 2007;164: 797-803.

57. Mannelli P, Peindl KS, Lee T, et al. Buprenorphine-mediated transition from opioid agonist to antagonist treatment: state of the art and new perspectives. Curr Drug Abuse Rev 2012;5:52-63.

58. Center for Substance Abuse Treatment. Clinical guidelines for the use of buprenorphine in the treatment of opioid addiction. Treatment improvement protocol (TIP) Series 40. DHHS Publication No. (SMA) 04-3939. Rockville (MD): Substance Abuse and Mental Health Services Administration; 2004.

59. Ferri M, Minozzi S, Bo A, et al. Slow-release oral morphine as maintenance therapy for opioid dependence. Cochrane Database Syst Rev 2013;(6): CD009879.

60. Beck T, Haasen C, Verthein U, et al. Maintenance treatment for opioid dependence with slow-release oral morphine: a randomized cross-over, non-inferiority study versus methadone. Addiction 2014;109:617-26.

61. Falcato L, Beck T, Reimer J, et al. Self-reported cravings for heroin and cocaine during maintenance treatment with slow-release oral morphine compared with methadone: a randomized, crossover clinical trial. J Clin Psychopharmacol 2015;35:150-7.

62. Verthein U, Beck T, Haasen C, et al. Mental symptoms and drug use in maintenance treatment with slow-release oral morphine compared to methadone: results of a randomized crossover study. Eur Addict Res 2015;21:97-104.

63. Hämmig R, Kohler W, Bonorden-Kleij K, et al. Safety and tolerability of slowrelease oral morphine versus methadone in the treatment of opioid dependence. J Subst Abuse Treat 2014;47:275-81.

64. New Information: Change of listing status of Kadian - open benefit to limited use. Non-Insured Health Benefits Program Newsletter 2014 Fall. Available: http://health.chiefs-of-ontario.org/sites/default/files/files/Health Canada NIHB Pharmacy Newsletter Fall 2014.pdf (accessed 2017 Mar. 10). 
65. Jegu J, Gallini A, Soler P, et al. Slow-release oral morphine for opioid maintenance treatment: a systematic review. Br J Clin Pharmacol 2011;71:832-43.

66. Tuten M, DeFulio A, Jones HE, et al. Abstinence-contingent recovery housing and reinforcement-based treatment following opioid detoxification. Addiction 2012;107:973-82.

67. Luty J. What works in drug addiction? Adv Psychiatr Treat 2003;9:280-8.

68. Strang J, McCambridge J, Best D, et al. Loss of tolerance and overdose mortality after inpatient opiate detoxification: follow up study. BMJ 2003;326:959-60.

69. Amato L, Davoli M, Minozzi S, et al. Methadone at tapered doses for the management of opioid withdrawal. Cochrane Database Syst Rev 2013;(2):CD003409.

70. Gowing L, Farrell M, Ali R, et al. Alpha(2)-adrenergic agonists for the management of opioid withdrawal. Cochrane Database Syst Rev 2016;(5):CD002024.

71. Gowing L, Ali R, White JM, et al. Buprenorphine for managing opioid withdrawal. Cochrane Database Syst Rev 2017;(2):CD002025.

72. Minozzi S, Amato L, Vecchi S, et al. Oral naltrexone maintenance treatment for opioid dependence. Cochrane Database Syst Rev 2011;(4):CD001333.

73. Amato L, Minozzi S, Davoli M, et al. Psychosocial combined with agonist maintenance treatments versus agonist maintenance treatments alone for treatment of opioid dependence. Cochrane Database Syst Rev 2011;(10):CD004147.

74. American Psychiatric Association. Diagnostic and statistical manual of mental disorders. Fifth edition. Arlington (VA): American Psychiatric Association Publishing; 2013.

75. Minozzi S, Amato L, Bellisario C, et al. Maintenance agonist treatments for opiatedependent pregnant women. Cochrane Database Syst Rev 2013;(12):CD006318.

76. Jones HE, Martin PR, Heil SH, et al. Treatment of opioid-dependent pregnant women: clinical and research issues. J Subst Abuse Treat 2008;35:245-59.

77. Lund IO, Fitzsimons $\mathrm{H}$, Tuten M, et al. Comparing methadone and buprenorphine maintenance with methadone-assisted withdrawal for the treatment of opioid dependence during pregnancy: maternal and neonatal outcomes. Subst Abuse Rehabil 2012;3(Suppl 1):17-25.

78. Zedler BK, Mann AL, Kim MM, et al. Buprenorphine compared with methadone to treat pregnant women with opioid use disorder: a systematic review and metaanalysis of safety in the mother, fetus and child. Addiction 2016;111:2115-28.
79. Noormohammadi A, Forinash A, Yancey A, et al. Buprenorphine versus methadone for opioid dependence in pregnancy. Ann Pharmacother 2016;50:666-72.

80. McCarthy JJ, Leamon MH, Finnegan LP, et al. Opioid dependence and preg nancy: minimizing stress on the fetal brain. Am J Obstet Gynecol 2017;216: 226-31.

81. Smyth BP, Barry J, Keenan E, et al. Lapse and relapse following inpatient treatment of opiate dependence. Ir Med J 2010;103:176-9.

82. Gossop M, Stewart D, Browne N, et al. Factors associated with abstinence, lapse or relapse to heroin use after residential treatment: protective effect of coping responses. Addiction 2002;97:1259-67.

83. Chen T, Masson CL, Sorensen JL, et al. Residential treatment modifications: adjunctive services to accommodate clients on methadone. Am J Drug Alcohol Abuse 2009;35:91-4.

84. Greenberg B, Hall DH, Sorensen JL. Methadone maintenance therapy in residential therapeutic community settings: challenges and promise. J Psychoactive Drugs 2007;39:203-10.

85. Sorensen JL, Andrews S, Delucchi KL, et al. Methadone patients in the therapeutic community: a test of equivalency. Drug Alcohol Depend 2009;100:100-6.

86. Kampman K, Jarvis M. American Society of Addiction Medicine (ASAM) National Practice Guideline for the use of medications in the treatment of addiction involving opioid use. J Addict Med 2015;9:358-67.

87. Guidelines for the psychosocially assisted pharmacological treatment of opioid dependence. Geneva: World Health Organization; 2009. Available: https:// www.ncbi.nlm.nih.gov/books/NBK143185 (accessed 2017 June 27).

88. Weiss RD, Potter JS, Griffin ML, et al. Long-term outcomes from the National Drug Abuse Treatment Clinical Trials Network Prescription Opioid Addiction Treatment Study. Drug Alcohol Depend 2015;150:112-9.

89. Weiss RD, Potter JS, Fiellin DA, et al. Adjunctive counseling during brief and extended buprenorphine-naloxone treatment for prescription opioid dependence: a 2-phase randomized controlled trial. Arch Gen Psychiatry 2011;68:1238-46.

90. Fiellin DA, Schottenfeld RS, Cutter CJ, et al. Primary care-based buprenorphine taper vs maintenance therapy for prescription opioid dependence: a randomized clinical trial. JAMA Intern Med 2014;174:1947-54.
Competing interests: Julie Bruneau, Benedikt Fischer, T. Cameron Wild, and Evan Wood report grants from the Canadian Institutes of Health Research (CIHR) during the conduct of the study. Peter Selby reports grants from $\mathrm{ClHR}$ to the Centre for Addiction and Mental Health during the conduct of the study. Peter Selby conducted an expert review for the Canadian Agency for Drugs and Technologies in Health on a rapid analysis for a formulation of buprenorphine in the last 12 months. Marie-Ėve Goyer reports payments from Indivior, Gilead Sciences, ViiV Healthcare and Merck, outside of the submitted work. Julie Bruneau reports monetary compensation as advisor from Gilead and Merck, outside of the submitted work. No other competing interests were declared.

This article has been peer reviewed.

Affiliations: Centre hospitalier de l'Université de Montréal (Bruneau), Université de Montréal; Département de médecine de famille et de médecine d'urgence (Bruneau, Goyer), Faculté de médecine, Université de Montréal, Montréal, Que.; British Columbia Centre on Substance Use (Ahamad), St. Paul's Hospital; Department of Family Medicine (Ahamad), University of British Columbia, Vancouver, BC; Centre de recherche et d'aide pour narcomanes, Centre intégré universitaire de santé et de services sociaux du Centre-Sud-de-l'île-de-
Montréal (Goyer), Montréal, Que.; Addictions Foundation of Manitoba (Poulin); Max Rady College of Medicine, Postgraduate Medical Education (Poulin), Rady Faculty of Health Sciences, University of Manitoba, Winnipeg, Man.; Addictions Division (Selby), Centre for Addiction and Mental Health; Department of Family and Community Medicine, Dalla Lana School of Public Health (Selby); Department of Psychiatry (Selby, Fischer), University of Toronto, Toronto, Ont.; Institute for Mental Health Policy Research (Fischer), Centre for Addiction and Mental Health, Toronto, Ont.; School of Public Health (Wild), Edmonton Clinic Health Academy, University of Alberta, Edmonton, Alta.; British Columbia Centre on Substance Use (Wood), St. Paul's Hospital; Department of Medicine (Wood), University of British Columbia, Vancouver, BC

Contributors: The four nominated principal investigators of the Canadian Research initiative in Substance Misuse (Julie Bruneau, Benedikt Fischer, T. Cameron Wild and Evan Wood) structured and coordinated the development process of the guideline, including the identification of the clinical leads. Clinical leads (Keith Ahamad, Marie-Ève Goyer, Ginette Poulin and Peter Selby) coordinated consultations and revision activities of the pan-Canadian review committee. Julie Bruneau and Evan Wood wrote the first version of the manuscript. All authors were actively involved in the various cycles of corrections and revisions of the manuscript, and contributed substantially to the interpretation of the findings. All of the authors gave final approval of the version to be published and agreed to act as guarantors of the work.

Acknowledgements: The authors are thankful to all committee members for their involvement as clinical experts and reviewers, as well as to Emily Wagner, Maryam Babaei, Annie Tremblay, Pauline Voon, Nirupa Goel, Aissata Sako, and all the Canadian Research Initiative in Substance Misuse staff involved in the production of this guideline.

Funding: This work was undertaken, in part, thanks to funding from the Canada Research Chairs Program through a Tier One Canada Research Chair in Inner City Medicine, which supports Evan Wood. Keith Ahamad is sup ported by a Canadian Institutes of Health Research (CIHR) Embedded Clinician Researcher Salary Award (TIC-368775). Benedikt Fischer acknowledges research support from the Chair in Addiction, Department of Psychiatry, University of Toronto. The four nodes of the Canadian Research Initiative in Substance Misuse received independent funding through a CIHR prioritydriven initiative (British Columbia: SMN-139148; the Prairies: SMN-139149; Ontario: SMN-139150; Quebec-Atlantic: SMN-139151).

Correspondence to: Julie Bruneau, julie. bruneau@umontreal.ca 